Revista de Matemática: Teoría y Aplicaciones 2009 16(2): 205-220

CIMPA - UCR ISSN: 1409-2433

\title{
PORTFOLIO OPTIMIZATION USING PARTICLE SWARMS WITH STRIPES
}

\author{
MARIO Villalobos-ARIAS*
}

Recibido/Received: 2 Oct 2008 - Aceptado/Accepted: 5 Mar 2009

\begin{abstract}
In this paper it is consider the Portfolio Optimization Problem developed by Markowitz [11]. The basic assumption is that the investor tries to maximize his/her profit and at the same time, wants to minimize the risk. This problem is usually solved using a scalarization approach (with one objective). Here it is solved it as a bi-objective optimization problem. It uses a new version of the algorithm of Particle Swarm Optimization for Multi-Objective Problems to which it implemented a method of the stripes to improve dispersion.
\end{abstract}

Keywords: Portfolio optimization, particle swarm optimization, multiobjetive, optimization.

\section{Resumen}

En el presente trabajo se considera el problema de optimización de portafolios desarrollado por Markowitz [11]. El supuesto básico es que el inversor intenta maximizar sus beneficios y al mismo tiempo, quiere minimizar el riesgo. Este problema se suele resolver mediante un enfoque de esscalarización (con un objetivo). Aquí se resuelve como un problema de optimización multiobjetivo. Utiliza una nueva versión del algoritmo de optimización por enjambre de partículas para problemas multiobjetivo, a los que se puso en práctica un método de las franjas para mejorar la dispersión.

Palabras clave: Optimización de portafolios, optimización por enjambre de partículas, multiobjetivo, optimización.

Mathematics Subject Classification: 90C27, 90C29, 90C59, 91B28.

\footnotetext{
${ }^{*}$ CIMPA, Escuela de Matemática, Universidad de Costa Rica 2060 San José, Costa Rica. E-Mail: mario.villalobos@ucr.ac.cr
} 


\section{Introduction}

In real-world, there are many problems with several objectives that we aim to optimize simultaneously, an example is the Portfolio Optimization, in wich the investor want to maximize his/her profit and the same time minimize the risk.

These problems are called "multiobjective" or "vector" optimization problems, and have been studied by many authors who have proposed a number of solution techniques $[1,4$, $6,12,13]$.

The solution of a multiobjective optimization problem requires a suitable definition of "optimality" (usually called "Pareto optimality"). Such problems normally have not one, but an infinite set of solutions, which represent possible trade-offs among the objectives (such solutions constitute the so-called "Pareto optimal set", defined in Section 2).

In these multiobjective optimization problems (MOPs) one wishes to optimize a vector function, say $F(x)=\left(f_{1}(x), \ldots, f_{n}(x)\right)$. A typical way to approach these problems is to transform the MOPs into single-objective (or "scalar") problems (e.g., by using a linear aggregating function). This approach indeed makes sense if the functions $f_{1}, \ldots, f_{n}$ are of the same type and expressed in the same units, but otherwise (for instance, if $f_{1}$ denotes distance, $f_{2}$ denotes time, and so on) the scalarized problem might be meaningless.

Diverse metaheuristics have been adopted to solve MOP [1]-[3], [5, 7], hence it is reasonable use a heuristic, such as particle swarm optimization (PSO), to solve the portfolio optimization problem as a multiobjective problem. In this paper we use the Particle Swarm Optimization algorithm for multiobjective (MOPSO) [2], and we use the stripes approach to improve this algorithm [14].

The rest of the paper is organized as follow, the next section MOP is presented, in section 3 an introduction to PSO is presented. The Portfolio Optimization Problem (POP) is presented in the section 4, some classical solution of the POP are in the section 5 . The data that we use and the result obtained is presented in sections 6 and 7. Finally in section 8 the conclusions and future work are presented.

\section{The multiobjective optimization problem}

Let $X$ be a set and $F: X \longrightarrow \mathbb{R}^{d}$ a given vector function with components $f_{i}: X \longrightarrow \mathbb{R}$ for each $i \in\{1, \ldots, d\}$. The multiobjective optimization problem (MOP) we are concerned with is to find $x^{*} \in X$ such that

$$
F\left(x^{*}\right)=\min _{x \in X} F(x)=\min _{x \in X}\left[f_{1}(x), \ldots, f_{d}(x)\right]
$$

where the minimum is understood in the sense of the standard Pareto order in which two vectors in $\mathbb{R}^{d}$ are compared as follows.

If $\vec{u}=\left(u_{1}, \ldots, u_{d}\right)$ and $\vec{v}=\left(v_{1}, \ldots, v_{d}\right)$ are vectors in $\mathbb{R}^{d}$, then

$$
\vec{u} \preceq \vec{v} \Longleftrightarrow u_{i} \leq v_{i} \forall i \in\{1, \ldots, d\}
$$




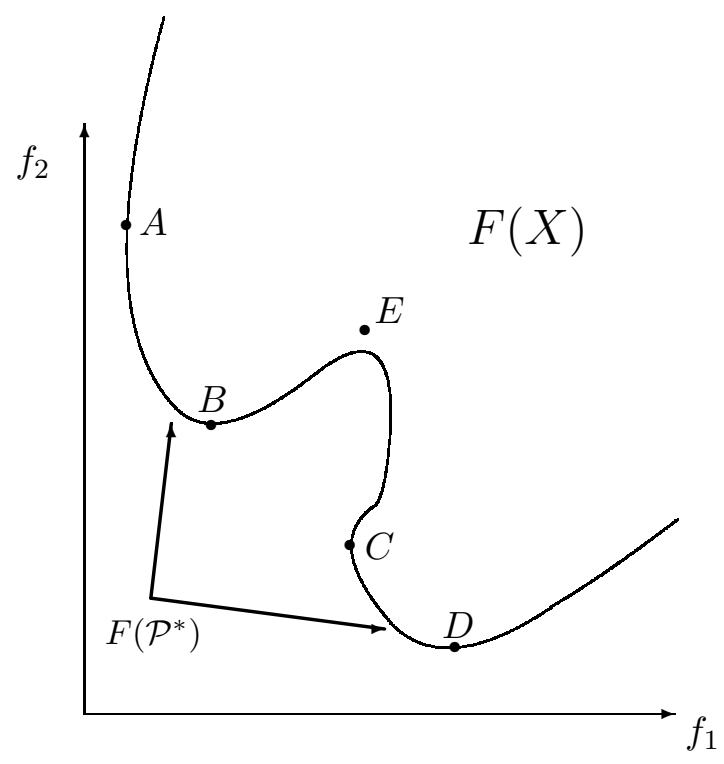

Figure 1: Example of a Pareto front for two objective case.

This relation is a partial order. We also write $\vec{u} \prec \vec{v}$ if $\vec{u} \preceq \vec{v}$ and $\vec{u} \neq \vec{v}$. In this case we say that $u$ dominates $v$. By example in Figure 1 point $B$ dominates point $E$.

Definition 1 A point $x^{*} \in X$ is called a Pareto optimal solution for the MOP (1) if there is no $x \in X$ such that $F(x) \prec F\left(x^{*}\right)$. The set

$$
\mathcal{P}^{*}=\{x \in X: x \text { is a Pareto optimal solution }\}
$$

is called the Pareto optimal set for the MOP (1), and its image under F, i.e.

$$
F\left(\mathcal{P}^{*}\right):=\left\{F(x): x \in \mathcal{P}^{*}\right\}
$$

is called the Pareto front.

In Figure 1 the Pareto front corresponds to the parts on the boundary of $F(X)$ joining the points $A$ and $B$, and also the points $C$ and $D$.

Here we say that $x$ dominates $y$ when $F(x) \prec F(y)$. Let $Y \subseteq X$ and $y \in Y$. If there is no $x \in Y$, that dominates $y$, we say that $y$ is nondominated (with respect to $Y$ ). Observe that all the elements in the Pareto front are nondominated with respect to $X$.

\section{Particle swarm optimization}

Particle swarm optimization algorithm (PSO) was introduced by Kennedy and Eberhart in 1995 [8] is based on the interaction of a set of particles that correspond to possible solutions of an optimization problem, moving each particle in a numerical space looking 
for the optimal position. A particularity of PSO is that particles communicate and hence - as in a social system - a particle with a good position (measured by its objective function value) influences on the other ones, attracting them.

In the PSO algorithm a set of $M$ particles is handled in a multidimensional space and it is intended to improve its performance according to its own experience and the experience of its neighbors. Indeed, each particle has three tendencies:

(i) to follow its present direction, following the particle's inertia,

(ii) to go back to its best historical position and

(iii) to imitate its best neighbor.

\subsection{Modeling PSO}

If $z^{m}(t)$ represents the $m$-th particle, then its velocity in iteration $t+1$ is defined as

$$
v^{m}(t+1)=\alpha v^{m}(t)+r_{1}\left(z^{m *}-z^{m}(t)\right)+r_{2}\left(z^{*}-z^{m}(t)\right)
$$

where $v^{m}(t)$ is the direction of the preceding iteration, $z^{m *}$ is the best historial position ever obtained by particle $m, z^{*}$ is the best particle ever obtained during the algorithm, $r_{1}$ and $r_{2}$ are random numbers, and $\alpha$ is a parameter. So, we define the new position of particle $m$ as

$$
z^{m}(t+1)=z^{m}(t)+v^{m}(t+1) .
$$

For more details about PSO see [8, 9] and for PSO for multiobjective problems see $[1,2]$.

\section{PSO with stripes}

To solve the portfolio optimization problem as a multiobjective problem we use a new approach presented in [14], call PSO with stripes (MOPSO-ST) that intent to solve the problem of the diversity of the MOP.

\section{The portfolio optimization problem: Markowitz model}

Here we present the Portfolio Optimization Problem developed by Markowitz [11]. The basic assumption is that the investor tries to maximize his/her profit and, at the same time, wants to minimize the risk.

We consider a market where $s$ different securities (i.e. stocks) are traded. These securities have prices $p_{1}, p_{2}, \ldots, p_{s}$ at the initial time $t=0$. We restrict ourselves to a one-period model. This means that the investor makes his decisions at the beginning of the period and is not allowed to revise his decisions until the end of the period. Let $P_{1}(T), P_{2}(T), \ldots, P_{s}(T)$ be the prices of the securities at the final time $t=T$, we assume that these final prices are not foreseeable. Therefore, they are modeled as non-negative random variables on a probability space $(\Omega, \mathcal{F}, \mathcal{P})$. 
The return of the stocks is given by the variables $r_{1}, r_{2}, \ldots, r_{s}$ given by

$$
r_{i}=\frac{P_{i}(T)-p_{i}}{p_{i}}, \quad i=1, \ldots, s .
$$

Observe that $r_{i}$ is also a random variable.

We assume that we know (or have estimated) their means, variances and covariances.

$$
\begin{aligned}
E\left(r_{i}\right) & =\mu_{i} \text { for all } i=1, \ldots, s, \\
\operatorname{Cov}\left(r_{i}, r_{j}\right) & =\sigma_{i j}^{2} \text { for all } i, j=1, \ldots, s .
\end{aligned}
$$

Using the variables $x_{i}$ for the share of the $i$-th security on the portfolio, we can calculate the return of the portfolio $R_{p}=R_{p}\left(x_{1}, \ldots, x_{s}\right)$ by

$$
R_{p}=\sum_{i=1}^{s} x_{i} r_{i}
$$

with the restrictions on the shares

$$
\sum_{i=1}^{s} x_{i}=1 \text { and } x_{i} \geq 0 \quad i=1, \ldots, s .
$$

We have observed that the $r_{i}$ are random variables with means $\mu_{i}$ and covariances $\sigma_{i j}^{2}=E\left(r_{i}-E\left(r_{i}\right)\right)\left(r_{j}-E\left(r_{j}\right)\right)$. Thus the return of the portfolio $R_{p}$ is a random variable as well, and its mean $\mu_{p}$ is given by

$$
\mu_{p}=E\left(R_{p}\right)=\sum_{i=1}^{s} x_{i} E\left(r_{i}\right)=\sum_{i=1}^{s} x_{i} \mu_{i}
$$

We measure the risk contained in the portfolio by the variance of its return

$$
\sigma_{p}^{2}=\operatorname{Var}\left(R_{p}\right)=E\left[\left\{R_{p}-E\left(R_{p}\right)\right\}^{2}\right]=\sum_{j=1}^{s} \sum_{i=1}^{s} x_{i} \sigma_{i j}^{2} x_{j}=\sum_{i, j=1}^{s} x_{i} x_{j} \sigma_{i j}^{2} .
$$

We will also impose the constraints

$$
x_{i} \leq c_{i}, \text { for all } i=1, \ldots, s,
$$

where the $c_{i}$ are constants.

Therefore, the investor wants to find a vector $\vec{x}=\left(x_{1}, x_{2}, \ldots, x_{s}\right)$ that maximizes the mean return

$$
\mu_{p}=\sum_{i=1}^{s} x_{i} \mu_{i}=:-f_{1}(\vec{x})
$$

and at the same time minimizes the risk

$$
\sigma_{p}^{2}=\sum_{i, j=1}^{s} x_{i} x_{j} \sigma_{i j}^{2}=: f_{2}(\vec{x})
$$


subject to the constraints

$$
\sum_{i=1}^{s} x_{i}=1 \text { and } 0 \leq x_{i} \leq c_{i} \forall i=1, \ldots, s .
$$

Thus, we have the next definition.

Definition 2 The classical portfolio optimization problem (POP) with two objective functions is to find the vector $\vec{x}^{*}=\left(x_{1}^{*}, x_{2}^{*}, \ldots, x_{s}^{*}\right)$ such that

$$
\begin{aligned}
\left(f_{1}\left(\vec{x}^{*}\right), f_{2}\left(\vec{x}^{*}\right)\right)= & \min _{\vec{x}}\left(-\sum_{i=1}^{s} x_{i} \mu_{i}, \sum_{i, j=1}^{s} x_{i} x_{j} \sigma_{i j}^{2}\right) \\
\text { subject to } \quad & \sum_{i=1}^{s} x_{i}=1, \\
& 0 \leq x_{i} \leq c_{i} \quad \forall i=1, \ldots, s .
\end{aligned}
$$

\section{Classical solution}

The classical way to solve this problem is by solving a single-objective (or scalar) problem, (see for example, [10]). One can also consider several variants of (5).

For instance we may require a lower bound $\left(R_{c}\right)$ on the mean return, and then choose the portfolio with minimal variance, that is

$$
\begin{aligned}
\min _{\vec{x}} \sigma_{p}^{2}= & \min _{\vec{x}} \sum_{i, j=1}^{s} x_{i} x_{j} \sigma_{i j}^{2} \\
\text { subject to } \quad & \mu_{p} \geq R_{c} \\
& \sum_{i=1}^{s} x_{i}=1, \\
& 0 \leq x_{i} \leq c_{i} \quad \forall i=1, \ldots, s
\end{aligned}
$$

Alternatively, one can consider the dual problem of setting up an upper bound $\left(\sigma_{c}\right)$ on the portfolio variance, and then maximize the mean return.

$$
\begin{aligned}
\max _{\vec{x}} \mu_{p}= & \max _{\vec{x}} \sum_{i=1}^{s} x_{i} \mu_{i} \\
\text { subject to } \quad & \sigma_{p}^{2} \leq \sigma_{c} \\
& \sum_{i=1}^{s} x_{i}=1, \\
& 0 \leq x_{i} \leq c_{i} \quad \forall i=1, \ldots, s .
\end{aligned}
$$


In any of these two forms of the POP, we usually find a single point of the Pareto front. (see Figure 2).

Still another variant of the POP is

$$
\begin{aligned}
\min _{\vec{x}}\left(\sigma_{p}^{2}-\mu_{p}\right)= & \min _{\vec{x}}\left(\sum_{i, j=1}^{s} x_{i} x_{j} \sigma_{i j}^{2}-\sum_{i=1}^{s} x_{i} \mu_{i}\right) \\
\text { subject to } \quad & \sum_{i=1}^{s} x_{i}=1, \\
& 0 \leq x_{i} \leq c_{i} \quad \forall i=1, \ldots, s .
\end{aligned}
$$

Again, the solution of this single-objective problem gives only one point of the Pareto front, and the investor does not have the option to select another portfolio with a similar risk and/or a better return.

This situation is illustrated in Figure 2, which shows a classical Pareto front for the POP. If the value of $\sigma_{c}$ is close to 0 , we can see that a small increase in the risk can give a much higher return. In contrast, if $\sigma_{p}^{2}$ is large, then to obtain a small increase in the return requires a large increase in the risk.

In the single-objective formulation of the POP, the investor cannot appreciate these subtleties.

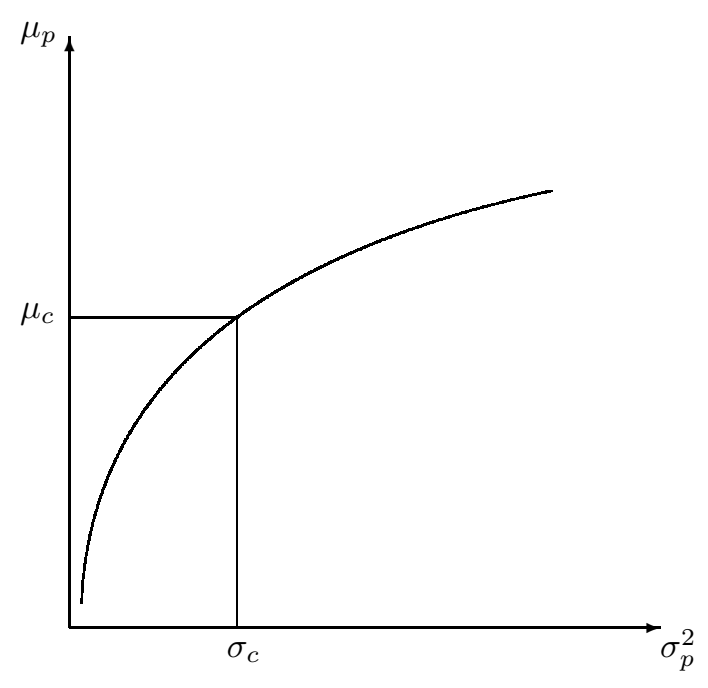

Figure 2: Graphical illustration of the Pareto front for the POP.

\section{The data}

To test our algorithm we took 20 securities (i.e. $s=20$ ) from the "Mexican Stock Market" $(\mathrm{BMV}=$ Bolsa Mexicana de Valores). These securities appear in the "Index of Prices and Quotations" (IPyC = Indice de Precios y Cotizaciones). 


\begin{tabular}{|c|c|c|c|c|c|c|c|c|c|}
\hline date & $\overline{\text { AlfaA }}$ & AmTelA1 & $\overline{\mathrm{Amxl}}$ & $\overline{\mathrm{BImboA}}$ & Cemex CPO & Elektra & Femsaubd & gcarsoa1 & $\cdots$ \\
\hline $28 / 09 / 2004$ & 42.090 & 23.800 & 22.027 & 24.752 & 63.800 & 76.200 & 50.200 & 51.843 & $\cdots$ \\
\hline $29 / 09 / 2004$ & 42.880 & 24.420 & 22.226 & 24.655 & 64.720 & 76.790 & 50.620 & 52.787 & $\cdots$ \\
\hline $30 / 09 / 2004$ & 43.060 & 24.600 & 22.206 & 24.439 & 64.090 & 76.480 & 50.300 & 51.992 & $\cdots$ \\
\hline $01 / 10 / 2004$ & 43.480 & 24.890 & 22.756 & 25.203 & 64.800 & 76.750 & 50.830 & 52.250 & $\ldots$ \\
\hline $04 / 10 / 2004$ & 43.280 & 25.250 & 23.185 & 25.350 & 65.760 & 76.400 & 50.870 & 52.558 & $\ldots$ \\
\hline $05 / 10 / 2004$ & 43.100 & 24.600 & 22.956 & 25.340 & 65.470 & 76.610 & 51.040 & 52.648 & $\cdots$ \\
\hline $06 / 10 / 2004$ & 42.860 & 24.300 & 22.526 & 25.144 & 67.140 & 76.690 & 51.140 & 52.518 & $\cdots$ \\
\hline $07 / 10 / 2004$ & 42.990 & 24.310 & 22.506 & 25.291 & 66.580 & 78.000 & 51.010 & 52.379 & $\cdots$ \\
\hline $08 / 10 / 2004$ & 42.150 & 23.810 & 22.007 & 24.214 & 65.120 & 79.500 & 50.920 & 52.131 & $\cdots$ \\
\hline$\vdots$ & : & $\vdots$ & $\vdots$ & : & $\vdots$ & $\vdots$ & : & : & $\ddots$ \\
\hline
\end{tabular}

Table 1: Example of table of prices.

\begin{tabular}{l|ccccccccc}
\hline \hline Fecha & AlfaA & AmTelA1 & Amxl & BImboA & Cemex CPO & Elektra & Femsaubd & gcarsoa1 & $\cdots$ \\
\hline $29 / 09 / 2004$ & 1.877 & 2.605 & 0.907 & -0.395 & 1.442 & 0.774 & 0.837 & 1.821 & $\cdots$ \\
$30 / 09 / 2004$ & 0.420 & 0.737 & -0.090 & -0.873 & -0.973 & -0.404 & -0.632 & -1.506 & $\cdots$ \\
$01 / 10 / 2004$ & 0.975 & 1.179 & 2.474 & 3.124 & 1.108 & 0.353 & 1.054 & 0.497 & $\cdots$ \\
$04 / 10 / 2004$ & -0.460 & 1.446 & 1.888 & 0.583 & 1.481 & -0.456 & 0.079 & 0.590 & $\cdots$ \\
$05 / 10 / 2004$ & -0.416 & -2.574 & -0.991 & -0.039 & -0.441 & 0.275 & 0.334 & 0.170 & $\cdots$ \\
$06 / 10 / 2004$ & -0.557 & -1.220 & -1.871 & -0.772 & 2.551 & 0.104 & 0.196 & -0.245 & $\cdots$ \\
$07 / 10 / 2004$ & 0.303 & 0.041 & -0.089 & 0.584 & -0.834 & 1.708 & -0.254 & -0.265 & $\cdots$ \\
$08 / 10 / 2004$ & -1.954 & -2.057 & -2.219 & -4.257 & -2.193 & 1.923 & -0.176 & -0.474 & $\cdots$ \\
$\vdots$ & $\vdots$ & $\vdots$ & $\vdots$ & $\vdots$ & $\vdots$ & $\vdots$ & $\vdots$ & $\vdots$ & $\ddots$
\end{tabular}

Table 2: Example of table of returns.

We took the prices of the 20 stocks for 100 days, see Table 1; the whole data is in [14]. Then we calculated the return of each security, for each day, using equation (2). See Table 2 , in [14] are the whole data.

To compute estimates of the mean returns and the covariances in (3) we used 5-day moving averages, that is, using the data from day $n-4$ to day $n$ with $n=5,6, \ldots, 100$. This procedure gave us 95 matrices of order $21 \times 20$ whose first row are the mean returns. An example of these matrices appears in Table 3.

Then to these data we applied the ST-MOPSO algorithm, see [14], to obtain a Pareto front for each of the 95 matrices. In each case we used the constraints $c_{i}=0.2$ for all $i=1, \ldots, s$.

\section{The results}

To apply the results obtained in the previous section, the idea was to use the 5-day data to decide the portfolio for the sixth day. Hence for each of the 95 matrices we tried to obtain a Pareto front with 100 points. The Table 4 is an example of the resulting solution, and Figure 3 shows the corresponding graph. (Appendix B contains all the graphs). The solutions tell us, according to the POP, the fraction (or the share (in percent)) of our 


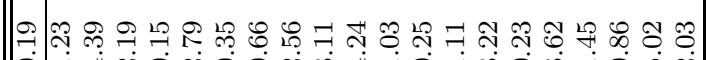

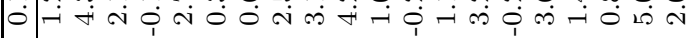

고응

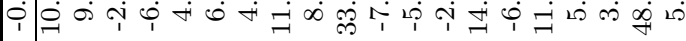

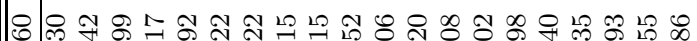

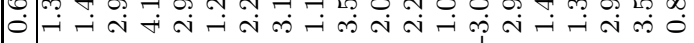

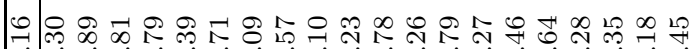

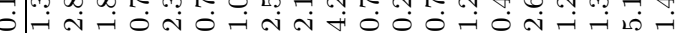

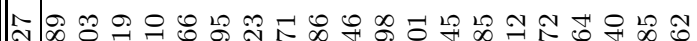

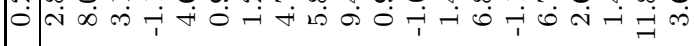

$\vec{\infty}$ 넌 -

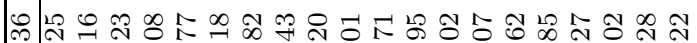

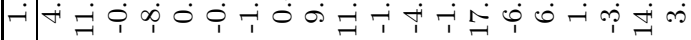

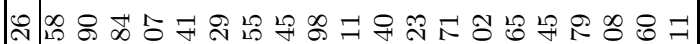

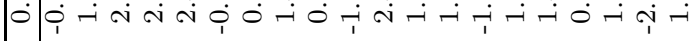

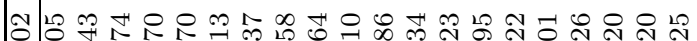

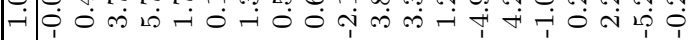

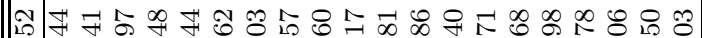

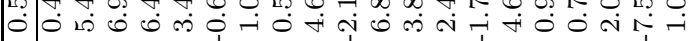

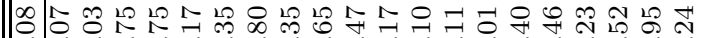
¡ं

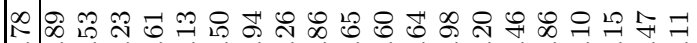

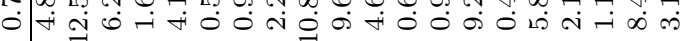

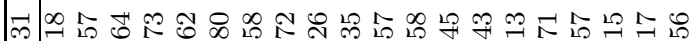
舟 冈 O- -

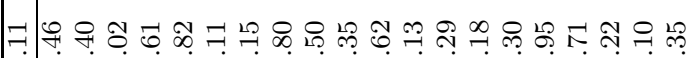

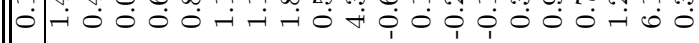

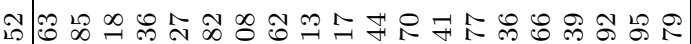

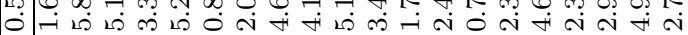

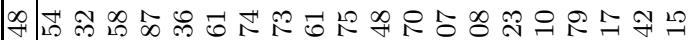

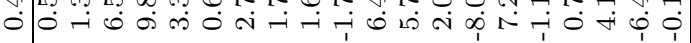

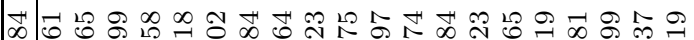
O- -

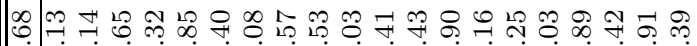

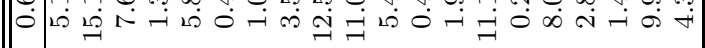

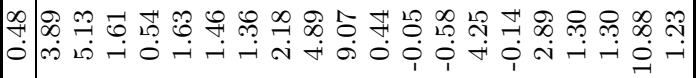




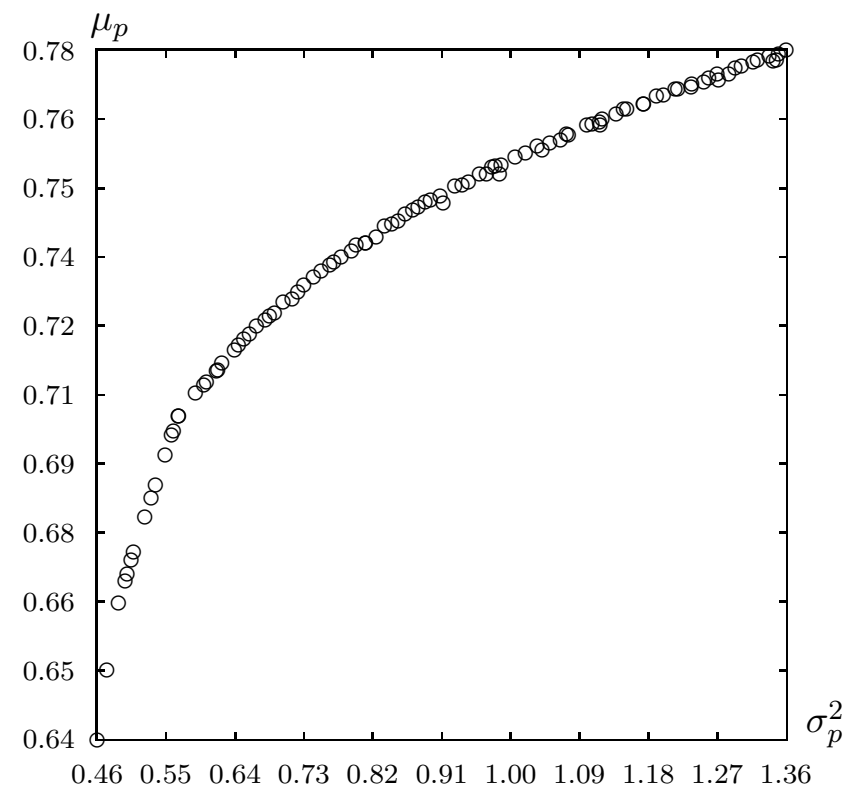

Figure 3: Example of the graph of a solution of POP.

wealth that we should invest in each of the 20 securities.

Since each of the 100 points in the Pareto front is a possible portfolio, handling this information turns out to be quite complicated. Therefore, we sorted the solutions according to their risks and took only three solutions per day: the solution with the minimal risk, the solution with the maximal risk, and a solution with a medium risk. Then we computed the return of for day 6 of these 3 solutions using equation 4, and we compared the return of these three solutions with the return of the IPyC of the BMV. The results are shown in Table 5 .

To see how this would work in a real situation, we did an experiment beginning with "one unit" of investment (say, one peso) and following the corresponding wealth day-perday; that is, every day we multiply the current value by $1+r$ to obtain the value of our investment the day after. The results are shown in the Table 6 and their graphical representation appears in Figure 4. It can be seen that each of our three solutions gives a better return than the IPyC-in some cases the return is up to $8 \%$ above the IPyC return (day 72 of Table 6). Only in the last few days our solutions were similar to the IPyCperhaps because the IPyC was behaving "optimally". For instance, from the Table 6.4 we can see that the IPyC return of $21.1 \%$ is very close to our solutions with minimal and maximum risks, $20.7 \%$ and $21.0 \%$, respectively, but below the $24.6 \%$ given by our medium risk solution. 


\begin{tabular}{|c|c|c|c|c|c|c|c|c|c|}
\hline day & IPyC & $\begin{array}{r}\text { min. } \\
\text { risk }\end{array}$ & $\begin{array}{r}\text { med. } \\
\text { risk }\end{array}$ & $\begin{array}{r}\text { max. } \\
\text { risk }\end{array}$ & day & IPyC & $\begin{array}{r}\text { min. } \\
\text { risk }\end{array}$ & $\begin{array}{r}\text { med. } \\
\text { risk }\end{array}$ & $\begin{array}{r}\text { max. } \\
\text { risk }\end{array}$ \\
\hline 1 & $-0.24 \%$ & $0.48 \%$ & $0.23 \%$ & $-0.21 \%$ & 48 & $1.11 \%$ & $1.24 \%$ & $2.01 \%$ & $2.45 \%$ \\
\hline 2 & $0.06 \%$ & $0.70 \%$ & $0.37 \%$ & $0.25 \%$ & 49 & $0.24 \%$ & $-0.29 \%$ & $-0.20 \%$ & $-0.53 \%$ \\
\hline 3 & $-1.62 \%$ & $-0.81 \%$ & $-1.04 \%$ & $-1.39 \%$ & 50 & $1.13 \%$ & $0.81 \%$ & $0.52 \%$ & $0.25 \%$ \\
\hline 4 & $0.49 \%$ & $0.52 \%$ & $0.42 \%$ & $0.36 \%$ & 51 & $0.50 \%$ & $0.38 \%$ & $0.80 \%$ & $0.98 \%$ \\
\hline 5 & $0.43 \%$ & $0.09 \%$ & $0.09 \%$ & $-0.05 \%$ & 52 & $0.09 \%$ & $-0.02 \%$ & $-0.22 \%$ & $-0.51 \%$ \\
\hline 6 & $-0.74 \%$ & $-1.45 \%$ & $-1.47 \%$ & $-1.56 \%$ & 53 & $0.11 \%$ & $-0.23 \%$ & $-0.22 \%$ & $-0.13 \%$ \\
\hline 7 & $-0.61 \%$ & $-0.58 \%$ & $-0.75 \%$ & $-0.41 \%$ & 54 & $-0.05 \%$ & $0.19 \%$ & $-0.17 \%$ & $-0.41 \%$ \\
\hline 8 & $1.05 \%$ & $0.73 \%$ & $0.83 \%$ & $0.56 \%$ & 55 & $1.09 \%$ & $0.79 \%$ & $1.09 \%$ & $0.62 \%$ \\
\hline 9 & $0.58 \%$ & $0.30 \%$ & $0.39 \%$ & $0.77 \%$ & 56 & $0.48 \%$ & $0.55 \%$ & $1.21 \%$ & $1.26 \%$ \\
\hline 10 & $-0.29 \%$ & $-0.13 \%$ & $0.04 \%$ & $0.04 \%$ & 57 & $0.28 \%$ & $0.21 \%$ & $0.49 \%$ & $0.67 \%$ \\
\hline 11 & $0.48 \%$ & $1.12 \%$ & $1.16 \%$ & $1.34 \%$ & 58 & $0.42 \%$ & $0.26 \%$ & $0.22 \%$ & $0.07 \%$ \\
\hline 12 & $0.81 \%$ & $1.00 \%$ & $1.12 \%$ & $1.15 \%$ & 59 & $0.13 \%$ & $0.04 \%$ & $0.43 \%$ & $0.82 \%$ \\
\hline 13 & $0.53 \%$ & $0.32 \%$ & $0.59 \%$ & $0.33 \%$ & 60 & $0.72 \%$ & $0.80 \%$ & $0.53 \%$ & $0.51 \%$ \\
\hline 14 & $-0.43 \%$ & $0.28 \%$ & $-0.05 \%$ & $0.19 \%$ & 61 & $0.91 \%$ & $0.65 \%$ & $0.88 \%$ & $0.85 \%$ \\
\hline 15 & $1.44 \%$ & $1.29 \%$ & $2.00 \%$ & $1.94 \%$ & 62 & $-0.48 \%$ & $-0.60 \%$ & $-0.58 \%$ & $-0.68 \%$ \\
\hline 16 & $1.53 \%$ & $0.53 \%$ & $0.17 \%$ & $0.37 \%$ & 63 & $-0.39 \%$ & $-0.22 \%$ & $-0.22 \%$ & $-0.18 \%$ \\
\hline 17 & $-0.66 \%$ & $-0.11 \%$ & $-0.69 \%$ & $-1.38 \%$ & 64 & $0.81 \%$ & $-0.41 \%$ & $0.35 \%$ & $0.21 \%$ \\
\hline 18 & $1.05 \%$ & $0.77 \%$ & $0.90 \%$ & $1.38 \%$ & 65 & $-1.92 \%$ & $-1.66 \%$ & $-1.65 \%$ & $-2.47 \%$ \\
\hline 19 & $0.50 \%$ & $0.41 \%$ & $0.43 \%$ & $-0.09 \%$ & 66 & $-1.48 \%$ & $-1.01 \%$ & $-1.47 \%$ & $-1.57 \%$ \\
\hline 20 & $-0.10 \%$ & $-0.01 \%$ & $-0.10 \%$ & $-0.05 \%$ & 67 & $0.88 \%$ & $0.05 \%$ & $0.28 \%$ & $1.28 \%$ \\
\hline 21 & $1.32 \%$ & $1.06 \%$ & $1.61 \%$ & $1.43 \%$ & 68 & $-2.01 \%$ & $-1.16 \%$ & $-1.10 \%$ & $-2.67 \%$ \\
\hline 22 & $0.83 \%$ & $0.72 \%$ & $0.85 \%$ & $0.96 \%$ & 69 & $-0.06 \%$ & $-0.45 \%$ & $-0.39 \%$ & $-0.49 \%$ \\
\hline 23 & $-0.60 \%$ & $-0.50 \%$ & $-0.29 \%$ & $-0.61 \%$ & 70 & $-1.88 \%$ & $-0.58 \%$ & $-0.44 \%$ & $-0.56 \%$ \\
\hline 24 & $-0.29 \%$ & $0.00 \%$ & $0.05 \%$ & $-0.80 \%$ & 71 & $0.91 \%$ & $0.57 \%$ & $0.76 \%$ & $0.83 \%$ \\
\hline 25 & $0.23 \%$ & $1.05 \%$ & $0.11 \%$ & $0.47 \%$ & 72 & $1.07 \%$ & $1.38 \%$ & $1.22 \%$ & $1.19 \%$ \\
\hline 26 & $0.06 \%$ & $0.67 \%$ & $0.92 \%$ & $0.40 \%$ & 73 & $1.83 \%$ & $1.27 \%$ & $1.45 \%$ & $1.56 \%$ \\
\hline 27 & $1.49 \%$ & $0.19 \%$ & $0.64 \%$ & $1.14 \%$ & 74 & $0.96 \%$ & $0.70 \%$ & $0.61 \%$ & $0.61 \%$ \\
\hline 28 & $-0.12 \%$ & $-0.22 \%$ & $-0.52 \%$ & $-0.10 \%$ & 75 & $1.63 \%$ & $1.86 \%$ & $1.83 \%$ & $1.84 \%$ \\
\hline 29 & $-0.02 \%$ & $0.21 \%$ & $0.29 \%$ & $0.46 \%$ & 76 & $0.05 \%$ & $0.47 \%$ & $-0.01 \%$ & $0.38 \%$ \\
\hline 30 & $-0.04 \%$ & $0.05 \%$ & $0.12 \%$ & $0.05 \%$ & 77 & $-2.08 \%$ & $-1.65 \%$ & $-2.05 \%$ & $-2.00 \%$ \\
\hline 31 & $0.58 \%$ & $0.89 \%$ & $0.45 \%$ & $0.56 \%$ & 78 & $-0.76 \%$ & $-1.04 \%$ & $-1.20 \%$ & $-1.11 \%$ \\
\hline 32 & $0.15 \%$ & $0.82 \%$ & $1.01 \%$ & $0.96 \%$ & 79 & $0.55 \%$ & $0.57 \%$ & $0.66 \%$ & $0.82 \%$ \\
\hline 33 & $-1.69 \%$ & $-1.07 \%$ & $-1.44 \%$ & $-1.75 \%$ & 80 & $0.96 \%$ & $0.48 \%$ & $0.99 \%$ & $0.33 \%$ \\
\hline 34 & $0.34 \%$ & $0.32 \%$ & $0.42 \%$ & $0.46 \%$ & 81 & $1.37 \%$ & $0.98 \%$ & $0.75 \%$ & $1.26 \%$ \\
\hline 35 & $-0.03 \%$ & $-0.40 \%$ & $-0.10 \%$ & $0.90 \%$ & 82 & $-0.45 \%$ & $-0.43 \%$ & $-0.77 \%$ & $-0.75 \%$ \\
\hline 36 & $0.26 \%$ & $0.17 \%$ & $0.12 \%$ & $1.09 \%$ & 83 & $0.42 \%$ & $-0.14 \%$ & $0.23 \%$ & $0.25 \%$ \\
\hline 37 & $0.75 \%$ & $0.83 \%$ & $0.95 \%$ & $1.09 \%$ & 84 & $0.43 \%$ & $0.45 \%$ & $-0.03 \%$ & $-0.35 \%$ \\
\hline 38 & $0.65 \%$ & $1.07 \%$ & $1.15 \%$ & $1.21 \%$ & 85 & $1.82 \%$ & $1.12 \%$ & $1.31 \%$ & $1.30 \%$ \\
\hline 39 & $0.99 \%$ & $1.71 \%$ & $1.98 \%$ & $1.37 \%$ & 86 & $-0.01 \%$ & $-0.31 \%$ & $-0.02 \%$ & $0.02 \%$ \\
\hline 40 & $-0.78 \%$ & $-0.18 \%$ & $-0.71 \%$ & $-0.22 \%$ & 87 & $0.75 \%$ & $0.80 \%$ & $0.91 \%$ & $0.46 \%$ \\
\hline 41 & $1.07 \%$ & $1.30 \%$ & $1.57 \%$ & $1.33 \%$ & 88 & $0.05 \%$ & $-1.11 \%$ & $-0.88 \%$ & $-0.77 \%$ \\
\hline 42 & $-0.97 \%$ & $-0.66 \%$ & $-0.70 \%$ & $-0.69 \%$ & 89 & $0.22 \%$ & $1.17 \%$ & $1.06 \%$ & $0.86 \%$ \\
\hline 43 & $-0.05 \%$ & $-0.65 \%$ & $-0.29 \%$ & $-0.27 \%$ & 90 & $0.23 \%$ & $-0.08 \%$ & $-0.26 \%$ & $-0.33 \%$ \\
\hline 44 & $0.66 \%$ & $-0.31 \%$ & $0.28 \%$ & $0.19 \%$ & 91 & $1.13 \%$ & $0.46 \%$ & $0.53 \%$ & $0.72 \%$ \\
\hline 45 & $-0.59 \%$ & $-0.46 \%$ & $-0.20 \%$ & $-0.01 \%$ & 92 & $0.34 \%$ & $0.47 \%$ & $0.15 \%$ & $-0.14 \%$ \\
\hline 46 & $-0.04 \%$ & $0.65 \%$ & $0.54 \%$ & $0.15 \%$ & 93 & $0.04 \%$ & $-0.17 \%$ & $0.04 \%$ & $0.05 \%$ \\
\hline 47 & $0.09 \%$ & $0.27 \%$ & $0.59 \%$ & $0.52 \%$ & 94 & $-1.10 \%$ & $-1.11 \%$ & $-1.16 \%$ & $-1.12 \%$ \\
\hline
\end{tabular}

Table 5: Table of comparison of return of IPyC, the solution with minimal risk, a medium risk and maximal risk. 


\begin{tabular}{|c|c|c|c|c|c|c|c|c|c|}
\hline day & $\mathrm{IPyC}$ & $\begin{array}{r}\text { min. } \\
\text { risk }\end{array}$ & $\begin{array}{r}\text { med. } \\
\text { risk }\end{array}$ & $\begin{array}{r}\max . \\
\text { risk }\end{array}$ & day & IPyC & $\begin{array}{r}\min . \\
\text { risk }\end{array}$ & $\begin{array}{r}\text { med. } \\
\text { risk }\end{array}$ & $\begin{array}{r}\max . \\
\text { risk }\end{array}$ \\
\hline 0 & 1.000 & 1.000 & 1.000 & 1.000 & & & & & \\
\hline 1 & 0.998 & 1.005 & 1.002 & 0.998 & 48 & 1.100 & 1.152 & 1.172 & 1.174 \\
\hline 2 & 0.998 & 1.012 & 1.006 & 1.000 & 49 & 1.102 & 1.148 & 1.170 & 1.168 \\
\hline 3 & 0.982 & 1.004 & 0.996 & 0.987 & 50 & 1.115 & 1.158 & 1.176 & 1.171 \\
\hline 4 & 0.987 & 1.009 & 1.000 & 0.990 & 51 & 1.120 & 1.162 & 1.185 & 1.182 \\
\hline 5 & 0.991 & 1.010 & 1.001 & 0.990 & 52 & 1.121 & 1.162 & 1.183 & 1.176 \\
\hline 6 & 0.984 & 0.995 & 0.986 & 0.974 & 53 & 1.123 & 1.159 & 1.180 & 1.175 \\
\hline 7 & 0.978 & 0.989 & 0.978 & 0.970 & 54 & 1.122 & 1.162 & 1.178 & 1.170 \\
\hline 8 & 0.988 & 0.997 & 0.987 & 0.975 & 55 & 1.134 & 1.171 & 1.191 & 1.177 \\
\hline 9 & 0.994 & 1.000 & 0.991 & 0.983 & 56 & 1.140 & 1.177 & 1.205 & 1.192 \\
\hline 10 & 0.991 & 0.998 & 0.991 & 0.983 & 57 & 1.143 & 1.180 & 1.211 & 1.200 \\
\hline 11 & 0.996 & 1.010 & 1.002 & 0.997 & 58 & 1.148 & 1.183 & 1.214 & 1.201 \\
\hline 12 & 1.004 & 1.020 & 1.014 & 1.008 & 59 & 1.149 & 1.183 & 1.219 & 1.211 \\
\hline 13 & 1.009 & 1.023 & 1.020 & 1.011 & 60 & 1.158 & 1.193 & 1.225 & 1.217 \\
\hline 14 & 1.005 & 1.026 & 1.019 & 1.013 & 61 & 1.168 & 1.200 & 1.236 & 1.227 \\
\hline 15 & 1.019 & 1.039 & 1.040 & 1.033 & 62 & 1.163 & 1.193 & 1.229 & 1.219 \\
\hline 16 & 1.035 & 1.045 & 1.041 & 1.037 & 63 & 1.158 & 1.190 & 1.226 & 1.217 \\
\hline 17 & 1.028 & 1.044 & 1.034 & 1.023 & 64 & 1.167 & 1.186 & 1.231 & 1.219 \\
\hline 18 & 1.039 & 1.052 & 1.043 & 1.037 & 65 & 1.145 & 1.166 & 1.210 & 1.189 \\
\hline 19 & 1.044 & 1.056 & 1.048 & 1.036 & 66 & 1.128 & 1.154 & 1.193 & 1.170 \\
\hline 20 & 1.043 & 1.056 & 1.047 & 1.035 & 67 & 1.138 & 1.155 & 1.196 & 1.185 \\
\hline 21 & 1.057 & 1.067 & 1.064 & 1.050 & 68 & 1.115 & 1.141 & 1.183 & 1.154 \\
\hline 22 & 1.066 & 1.075 & 1.073 & 1.060 & 69 & 1.115 & 1.136 & 1.178 & 1.148 \\
\hline 23 & 1.059 & 1.069 & 1.070 & 1.054 & 70 & 1.094 & 1.130 & 1.173 & 1.142 \\
\hline 24 & 1.056 & 1.069 & 1.070 & 1.045 & 71 & 1.104 & 1.136 & 1.182 & 1.151 \\
\hline 25 & 1.059 & 1.081 & 1.071 & 1.050 & 72 & 1.115 & 1.152 & 1.196 & 1.165 \\
\hline 26 & 1.059 & 1.088 & 1.081 & 1.054 & 73 & 1.136 & 1.166 & 1.214 & 1.183 \\
\hline 27 & 1.075 & 1.090 & 1.088 & 1.066 & 74 & 1.147 & 1.175 & 1.221 & 1.190 \\
\hline 28 & 1.074 & 1.087 & 1.082 & 1.065 & 75 & 1.165 & 1.196 & 1.244 & 1.212 \\
\hline 29 & 1.073 & 1.090 & 1.086 & 1.070 & 76 & 1.166 & 1.202 & 1.243 & 1.217 \\
\hline 30 & 1.073 & 1.090 & 1.087 & 1.071 & 77 & 1.142 & 1.182 & 1.218 & 1.192 \\
\hline 31 & 1.079 & 1.100 & 1.092 & 1.077 & 78 & 1.133 & 1.170 & 1.203 & 1.179 \\
\hline 32 & 1.081 & 1.109 & 1.103 & 1.087 & 79 & 1.139 & 1.177 & 1.211 & 1.189 \\
\hline 33 & 1.063 & 1.097 & 1.087 & 1.068 & 80 & 1.150 & 1.182 & 1.223 & 1.193 \\
\hline 34 & 1.066 & 1.101 & 1.092 & 1.073 & 81 & 1.166 & 1.194 & 1.232 & 1.208 \\
\hline 35 & 1.066 & 1.096 & 1.090 & 1.082 & 82 & 1.161 & 1.189 & 1.223 & 1.199 \\
\hline 36 & 1.069 & 1.098 & 1.092 & 1.094 & 83 & 1.165 & 1.187 & 1.226 & 1.202 \\
\hline 37 & 1.077 & 1.107 & 1.102 & 1.106 & 84 & 1.170 & 1.192 & 1.225 & 1.198 \\
\hline 38 & 1.084 & 1.119 & 1.115 & 1.120 & 85 & 1.192 & 1.206 & 1.241 & 1.213 \\
\hline 39 & 1.094 & 1.138 & 1.137 & 1.135 & 86 & 1.192 & 1.202 & 1.241 & 1.214 \\
\hline 40 & 1.086 & 1.136 & 1.129 & 1.132 & 87 & 1.201 & 1.212 & 1.252 & 1.219 \\
\hline 41 & 1.098 & 1.151 & 1.147 & 1.147 & 88 & 1.201 & 1.198 & 1.241 & 1.210 \\
\hline 42 & 1.087 & 1.143 & 1.138 & 1.139 & 89 & 1.204 & 1.212 & 1.255 & 1.220 \\
\hline 43 & 1.086 & 1.136 & 1.135 & 1.136 & 90 & 1.207 & 1.211 & 1.251 & 1.216 \\
\hline 44 & 1.094 & 1.132 & 1.138 & 1.139 & 91 & 1.220 & 1.217 & 1.258 & 1.225 \\
\hline 45 & 1.087 & 1.127 & 1.136 & 1.138 & 92 & 1.224 & 1.222 & 1.260 & 1.223 \\
\hline 46 & 1.087 & 1.135 & 1.142 & 1.140 & 93 & 1.225 & 1.220 & 1.260 & 1.224 \\
\hline 47 & 1.088 & 1.138 & 1.149 & 1.146 & 94 & 1.211 & 1.207 & 1.246 & 1.210 \\
\hline
\end{tabular}

Table 6: Table of comparison of investment of IPyC, the solution with minimal risk, a medium risk and maximal risk. 


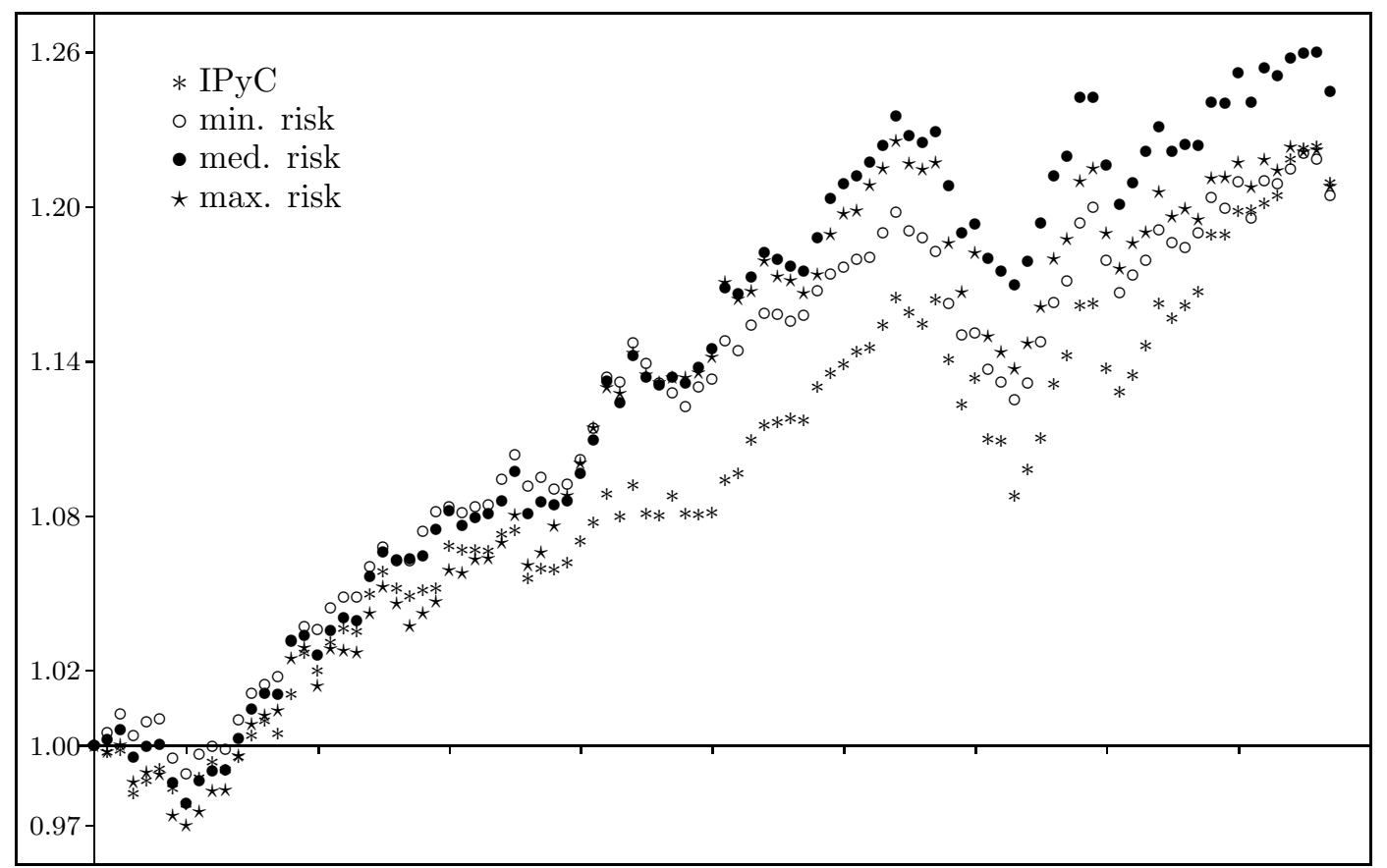

Figure 4: Graphic comparison of the IPyC and the solutions with minimal risk, a medium risk and maximal risk. 


\section{Conclusions and future work}

In this paper we applied the ST-MOPSO algorithm to the Markowitz' portfolio selection problem. As shown in section 7 our results seem to be quite good. But of course before reaching any conclusions we need to do more experimental work. For instance, we do not really know how good are our 5-day moving averages. It would be interesting (and important!) to determine how sensitive our results are to the length of the moving averages.

\section{References}

[1] Coello, C.A.; Van Veldhuizen, D.A.; Lamont, G.B.(2002) Evolutionary Algorithms for Solving Multi-Objective Problems. Kluwer Academic Publishers, New York.

[2] Coello, C.A.; Toscano, G.; Salazar, M. (2004) "Handling multiple objectives with particle swarm optimization", IEEE Transactions on Evolutionary Computation 8(3): $256-279$.

[3] Dasgupta, D. (1999) Artificial Immune Systems and Their Applications. SpringerVerlag, Berlin.

[4] Deb, K. (2001) Multi-Objective Optimization using Evolutionary Algorithms. John Wiley \& Sons, Chichester.

[5] Deb, K.; Pratap, A.; Agarwal, S.; Meyarivan, T. (2002) "A fast and elitist multiobjective genetic algorithm: NSGA-II", IEEE Transactions on Evolutionary Computation 6(2): $182-197$.

[6] Fonseca, C.M.; Fleming, P.J. (1997) "Multiobjective optimization", in: T. Bäck, D. B. Fogel, \& Z. Michalewicz (Eds.) Handbook of Evolutionary Computation, Oxford University Press: 1: C4.5:1-C4.5:9.

[7] Hui, X.; Eberhart, R.C.; Shi, Y. (2003) "Particle swarm with extended memory for multiobjective optimization", in: 2003 IEEE Swarm Intelligence Symposium Proceedings, IEEE Service Center, Indianapolis IN: 193-197.

[8] Kennedy, J.; Eberhart, R.C. (1995) "Particle swarm optimization", in: Proc. IEEE Int. Conf. Neural Networks, Piscataway NJ: 1942-1948.

[9] Kennedy, J.; Eberhart, R.C.(2001) Swarm Intelligence. Morgan Kaufmann Publishers, San Francisco CA.

[10] Korn, R. and Korn, E. (2000) Option Pricing and Portfolio Optimization: Modern Methods of Financial Mathematics, Graduate Studies in Mathematics 31. American Mathematical Society, Providence RI.

[11] Markowitz, H.M. (1952) "Portfolio selection", Journal of Finance 7(1):77-91. 
[12] Miettinen, K.M. (1998) Nonlinear Multiobjective Optimization. Kluwer Academic Publishers, Boston MS.

[13] Ulungu, E.L. (1993) Optimisation Combinatoire Multicritère: Determination de l'Ensemble des Solutions Efficaces et Méthodes Interactives. Ph.D. thesis, Faculté des Sciences, Université de Mons-Hainaut, Mons, Belgium.

[14] Villalobos-Arias, M. (2005) Análisis de Heurísticas de Optimización para Problemas Multiobjetivo. Ph.D. thesis, Departamento de Matemáticas, Cinvestav, México D.F., México. 